\title{
FUNDAMENTAL CYCLE OF A PERIODIC BOX-BALL SYSTEM: A NUMBER THEORETICAL ASPECT
}

\author{
TETSUJI TOKIHIRO and JUN MADA \\ Graduate school of Mathematical Sciences, \\ University of Tokyo, 3-8-1 Komaba, Tokyo 153-8914, Japan
}

(Received 6 November, 2003; accepted 31 March, 2004)

\begin{abstract}
A number theoretical aspect of the fundamental cycle of a periodic box-ball system is investigated. Using the formulae for the fundamental cycle of a class of initial states, we point out that the asymptotic behaviour of the fundamental cycle is closely related to the celebrated Riemann hypothesis.
\end{abstract}

2000 Mathematics Subject Classification. 37B15.

1. Introduction. The box-ball system (BBS) is a reinterpretation of a soliton cellular automaton proposed by Takahashi-Satsuma [1] as a dynamical system of balls in a one dimensional array of boxes [2]. It shows solitonic behaviour like $\mathrm{KdV}$ solitons and, in fact, is obtained from the discrete $\mathrm{KdV}$ equation through a limiting procedure called ultradiscretization [3]. Furthermore it is also a limit (crystallization) of a two dimensional integrable lattice model with the symmetry of the quantum algebra $U_{q}^{\prime}\left(A_{N}^{(1)}\right)[\mathbf{4}, \mathbf{5}, \mathbf{6}]$.

The periodic box-ball system (PBBS) is a BBS with a periodic boundary condition [7]. Like the BBS, the PBBS is obtained through both ultradiscretization and crystallization. For example, it is an ultradiscretization of the discrete Toda equation [8] with a periodic boundary condition and its initial value problem is solvable by combining the inverse scattering method and inverse ultradiscretization [9]. Hence we may call both the PBBS and the BBS integrable cellular automata.

An important difference between the BBS and the PBBS is that the PBBS can only take on a finite number of patterns and that the time evolution of the PBBS is necessarily periodic. Recently Yoshihara et al. have obtained the formulae that determine the fundamental cycle, i.e., the shortest period of the discrete periodic motion of the PBBS [10], and its asymptotic behaviour has been discussed by the authors [11]. In this article, using the results in [11], we will show that determining the asymptotic behaviour of the fundamental cycle of the PBBS for a certain type of initial state is equivalent to proving or disproving the Riemann hypothesis: all of the nontrivial zeros of $\zeta(s)$ lie on $\operatorname{Re}[s]=1 / 2$ [12]. Here $\zeta(s)$ is the Riemann-zeta function defined by $\zeta(s):=\sum_{n=1}^{\infty} \frac{1}{n^{s}}$. As is well known, the Riemann hypothesis is one of the most important unsolved problems in number theory, and it would be both interesting and worthwhile to point out a link between integrable cellular automata and number theory. In Section 2, we give the definition of the PBBS and briefly summarize the results concerning its fundamental cycle. Then, in Section 3, we show that a hypothesis on the fundamental cycle for a certain class of initial states is equivalent to the Riemann hypothesis. Section 4 is devoted to concluding remarks. 
2. Periodic Box-Ball system and its fundamental cycle. Let us consider a one dimensional array of $N$ boxes. To be able to impose a periodic boundary condition, we assume that the $N$ th box is adjacent to the first one. The box capacity is one for each box. We denote the number of balls by $M$, such that $M \leq \frac{N}{2}$. The balls are moved according to a deterministic time evolution rule. (See Figure 1.)

1. In each filled box, create a copy of the ball.

2. Move all the copies once according to the following rules.

3. Choose one of the copies and move it to the nearest empty box on the right of it.

4. Choose one of the remaining copies and move it to the nearest empty box on the right of it.

5. Repeat the above procedure until all the copies have moved.

6. Delete all the original balls.

A PBBS has conserved quantities which are characterized by a Young diagram with $M$ boxes. The Young diagram is constructed as follows. See also Figure 2. We denote an empty box by ' 0 ' and a filled box by ' 1 '. Then the PBBS is represented as a 0,1 sequence in which the last entry is regarded as adjacent to the first entry. Let $p_{1}$ be the number of the ' 10 ' pairs in the sequence. If we eliminate these ' 10 ' pairs, we obtain a new 0,1 sequence. We denote by $p_{2}$ the number of ' 10 ' pairs in the new sequence. We repeat the above procedure until all the ' 1 's are eliminated and obtain $p_{1}, p_{2}, p_{3}, \ldots, p_{l}$. Clearly $p_{1} \geq p_{2} \geq \cdots \geq p_{l}$ and $\sum_{i=1}^{l} p_{i}=M$. These $\left\{p_{i}\right\}_{i=1}^{l}$ are conserved in time. Since $\left\{p_{1}, p_{2}, \ldots, p_{l}\right\}$ is a weakly decreasing series of positive integers, we can associate it with a Young diagram with $p_{j}$ boxes in the $j$-th row $(j=1,2, \ldots, l)$. Then the lengths of the columns are also weakly decreasing positive integers, and we denote them

$$
\{\underbrace{L_{1}, L_{1}, \ldots, L_{1}}_{n_{1}}, \underbrace{L_{2}, L_{2}, \ldots, L_{2},}_{n_{2}} \cdots, \underbrace{L_{s}, L_{s}, \ldots, L_{s}}_{n_{s}}\}
$$

where $L_{1}>L_{2}>\cdots>L_{s}$. The set $\left\{L_{j}, n_{j}\right\}_{j=1}^{s}$ is an alternative expression of the conserved quantities of the system. In the limit $N \rightarrow \infty, L_{j}$ means the length of $j$ th largest soliton and $n_{j}$ is the number of solitons with length $L_{j}$.

The following proposition is essential in our arguments below. Let $\ell_{0}:=N-$ $2 M=N-\sum_{j=1}^{l} 2 p_{j}=N-\sum_{j=1}^{s} 2 n_{j} L_{j}, N_{0}:=\ell_{0}, L_{s+1}:=0$, and

$$
\begin{aligned}
& \ell_{j}:=L_{j}-L_{j+1} \quad(j=1,2, \ldots, s) \\
& N_{j}:=\ell_{0}+2 n_{1}\left(L_{1}-L_{j+1}\right)+2 n_{2}\left(L_{2}-L_{j+1}\right)+\cdots+2 n_{j}\left(L_{j}-L_{j+1}\right) \\
& =\ell_{0}+\sum_{k=1}^{j} 2 n_{k}\left(L_{k}-L_{j+1}\right) \text {. }
\end{aligned}
$$

Then, for a fixed number of boxes $N$ and conserved quantities $\left\{L_{j}, n_{j}\right\}$, the fundamental cycle $T$ is determined by the following proposition.

Proposition 1 (YYT). Let $\tilde{T}$ be defined by

$$
\tilde{T}:=\text { L.C.M. }\left(\frac{N_{s} N_{s-1}}{\ell_{s} \ell_{0}}, \frac{N_{s-1} N_{s-2}}{\ell_{s-1} \ell_{0}}, \cdots, \frac{N_{1} N_{0}}{\ell_{1} \ell_{0}}, 1\right),
$$




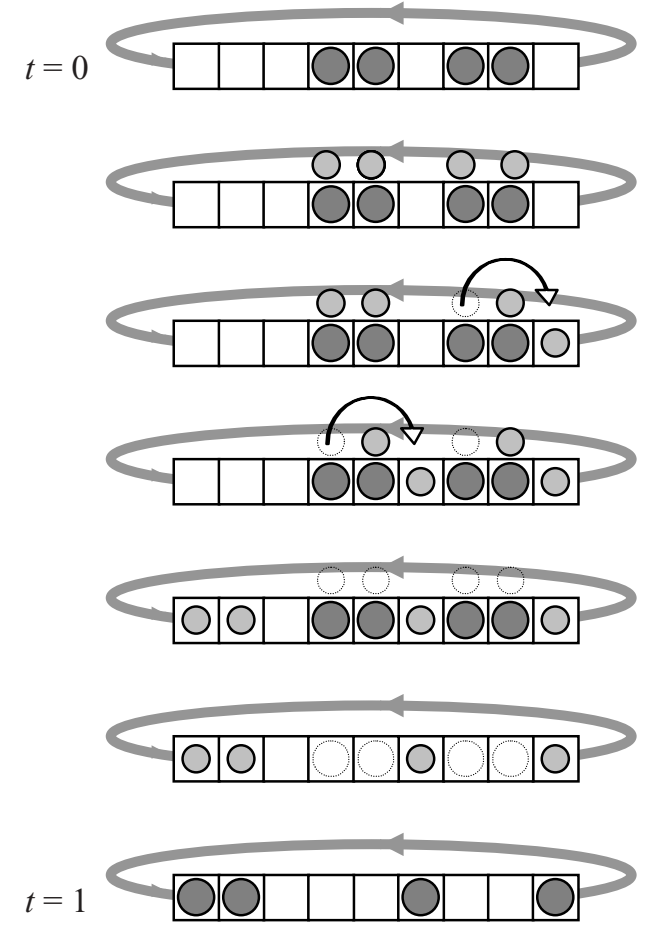

Figure 1. Time evolution rule for PBBS

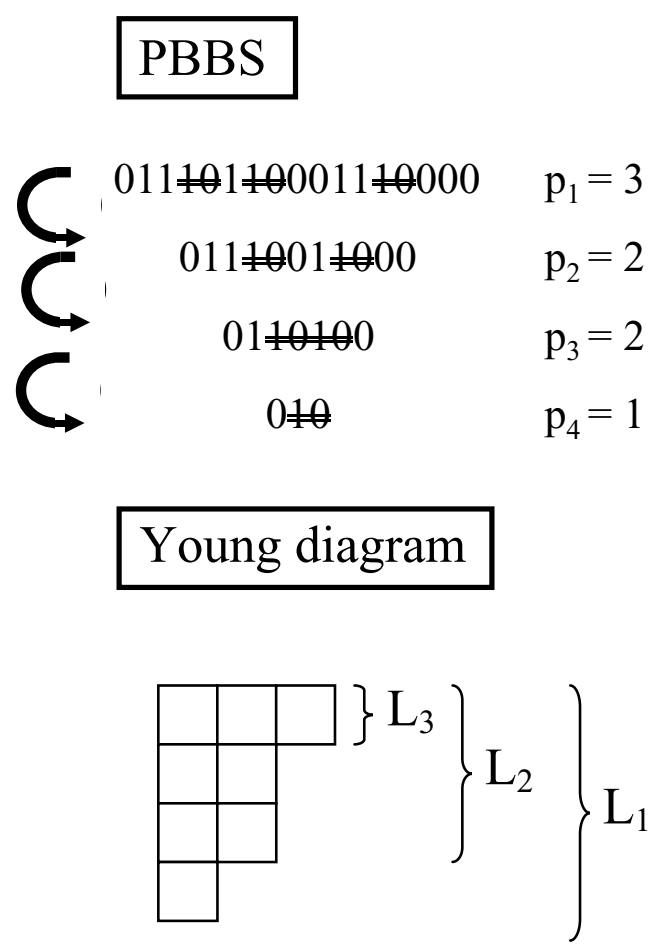

Figure 2. Correspondence between PBBS and Young diagram 
where L.C.M. $(x, y):=2^{\max \left[x_{2}, y_{2}\right]} 3^{\max \left[x_{3}, y_{3}\right]} 5^{\max \left[x_{5}, y_{5}\right]} \ldots$ for $x=2^{x_{2}} 3^{x_{3}} 5^{x_{5}} \ldots$ and $y=$ $2^{y_{2}} 3^{y_{3}} 5^{y_{5}} \ldots$. Then $T$ is a divisor of $\tilde{T}$. In particular, when there is no internal symmetry in the state, the fundamental cycle $T$ coincides with $\tilde{T}$.

The definition of internal symmetry in the above proposition is rather complicated and we refer to the original article [10]. However, for a given number of conserved quantities, we can always construct initial states which do not have any internal symmetry, in particular, if ${ }^{\forall} i, n_{i}=1$ the PBBS never has internal symmetry and $T=\tilde{T}$. A number theoretical aspect of the PBBS is suggested by the fact that $\tilde{T}$ is essentially given by the least common multiple of integers constructed from the conserved quantities.

3. Asymptotic behaviour of the fundamental cycle and the Riemann hypothesis. We would like to consider the asymptotic behaviour of the fundamental cycle for a class of initial states. To take an appropriate limit, we fix the ball density $\rho:=M / N$. We assume that $N$ is an even integer and $\rho$ is sufficiently large:

$$
\frac{2}{5}<\rho<\frac{9}{20}
$$

(These two assumptions are not essential in the arguments below, but they make the proof of our statements simpler.) Since $\ell_{0}=N-2 M, \ell_{0}$ is also an even integer. Let $k$ and $s$ be the integers which are determined uniquely for a given $N$ and $M$ by

$$
\begin{aligned}
k(k-1) & \leq \ell_{0} \leq k(k+1)-2 \\
(k+s-1)(k+s) & \leq N \leq(k+s)(k+s+1)-2
\end{aligned}
$$

Then we consider an initial state which consists of $s$ kinds of solitons with length $1,2, \ldots, s\left(\ell_{j}=1{ }^{\forall} j \geq 1\right)$. In view of (5) and (6), we choose the conserved quantities so that $n_{1}=\frac{k(k+1)-\ell_{0}}{2}, n_{2}=\frac{\ell_{0}-k(k-1)+2}{2}, n_{s}=\frac{N-(k+s)(k+s-1)+2}{2}$, and $n_{j}=1 \quad(3 \leq$ $j \leq s-1)$. By the definiton of $N_{j}(2)$, we have

$$
\begin{aligned}
N_{1} & =k(k+1), \quad N_{2}=(k+1)(k+2), \quad N_{3}=(k+2)(k+3), \ldots, \\
N_{s-1} & =(k+s-2)(k+s-1)\left(N_{s} \equiv N\right) .
\end{aligned}
$$

As was mentioned in the previous section, we can suppose that there is no internal symmetry in this state and its fundamental cycle $T(N)$ is estimated as

$$
\begin{aligned}
T_{\rho}(N)= & \text { L.C.M. }\left(\frac{N_{s} N_{s-1}}{\ell_{s} \ell_{0}}, \frac{N_{s-1} N_{s-2}}{\ell_{s-1} \ell_{0}}, \ldots, \frac{N_{2} N_{1}}{\ell_{1} \ell_{0}}, 1\right) \\
\geq & \frac{1}{\ell_{0}} \text { L.C.M. }\left(N_{s-1} N_{s-2}, N_{s-2} N_{s-3}, \ldots, N_{2} N_{1}\right) \\
= & \frac{1}{\ell_{0}} \text { L.C.M. }\left((k+s-1)(k+s-2)^{2}(k+s-3), \ldots,\right. \\
& \left.(k+3)(k+2)^{2}(k+1),(k+2)(k+1)^{2} k\right) \\
\geq & \frac{1}{\ell_{0}} \text { L.C.M. }\left((k+s-2)^{2},(k+s-3)^{2}, \ldots,(k+1)^{2}\right) \\
= & \frac{1}{\ell_{0}}(\text { L.C.M. }((k+s-2),(k+s-3), \ldots,(k+1)))^{2} .
\end{aligned}
$$


in a similar manner, we have

$$
\begin{aligned}
T_{\rho}(N) & \leq \text { L.C.M. }\left(N_{s} N_{s-1}, N_{s-1} N_{s-2}, \ldots, N_{2} N_{1}\right) \\
& \leq N(\text { L.C.M. }((k+s-1),(k+s-2), \ldots,(k+1), k))^{2} .
\end{aligned}
$$

For two positive integers $n$ and $m(n>m)$, we define

$$
L(n, m):=\text { L.C.M. }(n, n-1, \ldots, m+2, m+1) .
$$

Clearly $L(n, m) \leq L(n, 1)$ and $L(n, 1)=L(n, m)$ when $2 m \leq n$. From (7) and (8), we have

$$
\frac{(L(k+s-2, k))^{2}}{\ell_{0}} \leq T_{\rho}(N) \leq N(L(k+s-1, k-1))^{2}
$$

From (5) and (6), we have $\sqrt{N}-1<k+s<\sqrt{N}+1$ and $\sqrt{\ell_{0}}-1<k<\sqrt{\ell_{0}}+1$. Since $\ell_{0}=N-2 M<N / 5$ implies $2 k<k+s-2$ for sufficiently large $N$, the fundamental cycle is estimated as

$$
\frac{(L(k+s-2,1))^{2}}{\ell_{0}} \leq T_{\rho}(N) \leq N(L(k+s-1,1))^{2} .
$$

Now we introduce the Chebyshev function $\psi(n)$ [13]

$$
\psi(n):=\sum_{\substack{p, j \\ p^{j} \leq n}} \log p
$$

where the sum runs over all primes $p$ and $j \in \mathbb{Z}_{+}$such that $p^{j} \leq n$.

Then $L(n, 1)=$ L.C.M. $(n, n-1, n-2, \ldots, 2)$ is expressed as

$$
L(n, 1)=\exp [\psi(n)]
$$

and we obtain the inequality

$$
2 \psi(k+s-2)-\log \ell_{0} \leq \log T_{\rho}(N) \leq 2 \psi(k+s-1)+\log N
$$

The asymptotic formulae for the Chebyshev function $\psi(n)$ have been extensively investigated since the nineteen century in relation to the theory of prime numbers, and it is well known that the Riemann hypothesis is equivalent to the estimate [14]:

$$
\psi(x)=x+O\left(x^{1 / 2} \log ^{2} x\right) \quad(x \rightarrow+\infty) .
$$

Since $k+s=[\sqrt{N}]$ or $[\sqrt{N}]+1$ and $\log \ell_{0} \simeq 2 \log \sqrt{N}$ we found the following theorem.

THEOREM 1. The Riemann hypothesis is equivalent to the estimate

$$
\log T_{\rho}(N)=2 \sqrt{N}+O\left(N^{1 / 4} \log ^{2} N\right) \quad(N \rightarrow+\infty) .
$$

The following corollary is an immediate consequence of the Theorem 1. 
COROLlary 1. Let the maximum fundamental cycle for a given $N$ and $\rho$ which satisfy the inequality (4) be $T_{\rho}^{\operatorname{Max}}(N)$. Then the estimate

$$
\log T_{\rho}^{\operatorname{Max}}(N)=2 \sqrt{N}+O\left(N^{1 / 4} \log ^{2} N\right) \quad(N \rightarrow+\infty)
$$

implies the Riemann hypothesis.

As discussed in [11], we conjecture that $T_{\rho}(N) \simeq T_{\rho}^{\mathrm{Max}}(N)$, hence we think the above corollary has some significance.

4. Concluding remarks. We have shown a relation between the fundamental cycle of a PBBS and the Riemann hypothesis. Since the fundamental cycle is in principle obtained from the theta function solutions of the discrete Toda equation (through ultradiscretization), the investigation of the ultradiscretized period matrices may give some information about the Riemann hypothesis. Furthermore, as the eigenvalues of the transfer matrix of a particular two dimensional integrable lattice model also gives information about the fundamental cycle of the PBBS, it will be an important problem to construct an analogue of the Bethe anzatz for ultradiscrete systems.

Although there are many statements equivalent to the Riemann hypothesis [15] and Theorem 1 is only one of them, it reveals an important number theoretical aspect of the theory of integrable systems and we hope for new developments in this direction.

\section{REFERENCES}

1. D. Takahashi and J. Satsuma, A soliton cellular automaton, J. Phys. Soc. Japan. 59 (1990), 3514-3519.

2. D. Takahashi, On some soliton systems defined by using box and ball systems, in Proceedings of the International Symposium on Nonlinear Theory and Its Applications, NOLTA'93 (1991), 555-558.

3. T. Tokihiro, D. Takahashi, J. Matsukidaira and J. Satsuma, From soliton equations to integrable cellular automata through a limiting procedure, Phys. Rev. Lett. 76 (1996), 3247-3250.

4. K. Hikami, R. Inoue and Y. Komori, Crystallization of the Bogoyavlensky lattice, Journal of the Physical Society of Japan 68 (1999), 2234-2240.

5. K. Fukuda, M. Okado and Y. Yamada, Energy functions in box ball systems International Journal of Modern Physics A 15 (2000), 1379-1392.

6. G. Hatayama, K. Hikami, R. Inoue, A. Kuniba, T. Takagi, and T. Tokihiro, The $A_{M}^{(1)}$ automata related to crystals of symmetric tensors, J. Math. Phys. 42 (2001), 274.

7. F. Yura and T. Tokihrio, On a periodic soliton cellular automaton, J. Phys. AFMath. Gen. 35 (2002), 3787-3801.

8. R. Hirota, S. Tsujimoto and T. Imai, Difference Scheme of Soliton Equations, in Future Directions of Nonlinear Dynamics in Physics and Biological Systems (ed. by P. L. Christiansen, J. C. Eilbeck and R. D. Parmentier) Series B: Physics Vol. 312, Plenum (1992), 7-15.

9. T. Kimijima and T. Tokihiro, Initial-value problem of the discrete periodic Toda equation and its ultradiscretization, Inverse Problems 18 (2002), 1705-1732.

10. D. Yoshihara, F. Yura and T. Tokihiro, Fundamental Cycle of a Periodic Box-Ball System, J. Phys. AFMath. Gen. 36 (2003), 99-121.

11. J. Mada and T. Tokihiro, Asymptotic behaviour of fundamental cycle of periodic boxball systems, J. Phys. AFMath. Gen. 36 (2003), 7251-7268.

12. B. Riemann, Über die Anzahl der Primzahlen unter einer gegebener Grösse, Monatsber. Akad. Berlin (1859), 671-680.

13. See for example, D. J. Newman, Analytic number theory (Springer-Verlag, 1998).

14. M. R. Murty, Problems in analytic number theory (Springer-Verlag, 2001).

15. See for example, S. Kanemitsu and M. Yoshimoto, Farey series and the Riemann hypothesis, Acta Arith. 75 (1996), 351-374. 